\title{
THE PART PLAYED BY THE AMORPHOUS PHASE IN THE HARDENING OF STEELS.
}

Mr. J. C. W. Humfrey, B.A., M.Sc., M.Eng. (Sheffield), read a Paper on "The Part Played by the Amorphous Phase in the Hardening of Steels."

The existence in solid metals of an amorphous or undercooled liquid phase has already been deduced from two distinct points of view, viz. :-

I. As being formed in the crystal gliding planes when a metal is severely overstrained. This phenomenon has been particularly studied by Beilby.

2. As normally existing as a thin film or cement between the individual crystals of which a mass of metal is built up. This theory, which was originally independently advanced by several writers, has received strong experimental support from the work of Rosenhain and Ewen, and the present author. ${ }^{\mathrm{I}-4}$

In a paper ${ }^{4}$ dealing with the influence of the amorphous intercrystalline cement upon the mechanical properties of metals, the author put forward a suggestion that the recrystallization which occurs in iron and steel at the $\mathrm{Ar}_{\mathrm{I}}$ change-point does not take place directly, but by the preliminary breakdown of the $\gamma$ crystals to an amorphous state, followed by a subsequent formation of the $\beta$ or $a$ crystals from the amorphous. The suggestion was advanced in order to explain the continuous existence of an intercrystalline cement in iron irrespective of its allotropic changes. A somewhat similar idea was advanced many years ago by Barus and is quoted by Roberts-Austen in his Introduction to Metallurgy, as follows: "When iron passes through the temperature of re-calescence, its molecular condition is for an instant almost chaotic."

In the present paper the suggestion is examined further, and from it is deduced an explanation of the hardening of steels.

In the idea as originally advanced it was assumed that the amorphous cement which exists during the different allotropic ranges of iron is always of a similar nature, and corresponds in phase with that of the true liquid metal; further consideration of the matter has, however, led the author to a somewhat different view, and one, he thinks, in which the theory of the formation of an intermediate amorphous phase during recrystallization becomes more consistent with thermodynamical principles.

We know that a substance, in passing from the liquid to the solid (crystalline) form, changes from a state in which the units are irregularly dispersed to one in which they are arranged in some definite and regular manner, which is characteristic of the substance in question Numerous crystallographic researches-chiefly upon crystals having a perfect external form-have resulted in a theory of crystal structure which the author believes to be now generally accepted, and which has indeed received striking 
confirmation from the recent experiments upon the reflection of $\mathrm{X}$-rays from crystal surfaces. The theory in question asserts that the regularity of structure within a crystal is twofold, viz. :-

I. That the centres of gravity of the molecules are arranged together according to one of a series of geometrical devices called "space lattices," in which each point in the lattice is surrounded by a similar distribution of other points.

2. That in each molecule of a crystal the atoms are similarly situated.

The author considers that it is the second form of regularity which essentially gives rise to the first ; or in other words, that in crystallization from fusion it is the forces exerted by each molecule upon its neighbours (i.e. forces due to the resultant reactions of its atoms) which bring about the space-lattice structure of the crystal.

Now an allotropic change must be considered as being essentially accompanied by a change in the internal structure of the molecules, e.g. a reorganization of the atoms composing them or a change in their number. Thus we have the two gaseous allotropes of oxygen, the common gas in which each molecule contains two atoms, and ozone in which each molecule contains three atoms. When an allotropic change takes place in a crystalline body we must imagine a similar internal rearrangement of the atoms in each molecule ; and, if the new form which the molecules take involves a corresponding change in the external forces which they exert upon one another, then the previously existing space lattice may become unstable and a fresh one may be formed characteristic of some other crystal form. But before the reorganization can be completed there must, at least temporarily, be a state of disorder, and it is during this disorder that the author considers that the structure must be considered as amorphous. The intermediate amorphous state may be realized as corresponding to the liquid which would be formed by the fusion of the solid phase stable at the lower temperatures, if the conditions could be so adjusted that the subsequent recrystallization were avoided. Certain cases are known where such a phenomenon can be actually observed. Thus in the case of sulphur the change from the monoclinic to the rhombic and vice versa normally occurs at a temperature of $95^{\circ} 6^{\circ} \mathrm{C}$.; but the rhombic form stable below this temperature can be heated above it, and in this metastable condition melts at $115^{\circ} \mathrm{C}$; ; if, however, the change to monoclinic is allowed to complete itself before this temperature is reached, the monoclinic crystals of sulphur do not melt until $12 \mathrm{O}^{\circ} \mathrm{C}$.

In the case of iron the temperature-tenacity curves obtained by Rosenhain and the present author ${ }^{3}$ give strong indications that a similar phenomenon might occur, the rapid drop in the tenacity of both $\alpha$ and $\beta$ iron before $\mathrm{Ac}_{3}$ strongly suggesting that they are rapidly approaching their melting-points ; and that if on heating a sample of iron suitable conditions of pressure could be applied which prevented the recrystallization to the $\gamma$, then a true meltingpoint could be observed in the neighbourhood of $900^{\circ} \mathrm{C}$.

We know that physical changes of state, such as from liquid to solid or from one allotropic modification to another, do not take place instantaneously throughout the whole mass of a body, but that starting from certain nuclei they proceed gradually outwards. Thus we must conceive that the intermediate amorphous phase existing during an allotropic change which occurs normally (i.e. by reiatively slow heating or cooling) commences to recrystallize almost as soon as it is formed, and is present at any particular instant during the change merely as thin films or layers between the coexisting crystalline phases, the films moving forward as the change progresses. The course of the breakdown of the one crystalline phase to the amorphous.would tend to voL. $\mathrm{X}-\mathrm{T} 9$ 


\section{THE PART PLAYED BY THE AMORPHOUS PHASE}

follow those planes in which the freedom of movement was greatest, viz. the crystal gliding planes and possibly the intercrystalline boundaries.

That a second crystalline phase may form after the first has broken down to amorphous necessitates the condition that the mass is not too viscous for the forces of crystallization to overcome the viscosity and to marshal the molecules into their new orientation. We know from Beilby's work that such a condition is not invariably present, since he has shown that any amorphous phase formed by severe overstrain in the cold possesses a definite stability up to certain well-marked temperatures, and only passes back into the crystalline state when these temperatures are exceeded. Thus, if an allotropic change be made, by suitable conditions, to take place at a temperature at which an amorphous phase is stable, then we can conceive that while the breakdown from the one crystalline state may take place, yet the fresh crystallization may be prevented, and that under such conditions the amorphous phase formed by the breakdown will persist. That the breakdown from a crystalline to an amorphous state may take place in spite of low temperature is clearly shown by the formation of amorphous from the crystalline by overstrain, and also by the changes in certain alloy steels to be referred to later.

In a metal in which an allotropic change normally takes place at a temperature well above that at which the viscosity is sufficient to prevent crystallization, abnormal conditions-such as rapid cooling - may delay the change to well below this temperature.

From the general considerations which have been advanced above we may now pass to the particular case in view, viz. that of stecls. Firstly we have the following experimentally determined data :-

I. Pure iron undergoes an allotropic change at about $860^{\circ} \mathrm{C}$., which results in a complete recrystallization. The latter fact has been clearly proved by "heat-reliefs," 5 by the microscopical study of strain effects, ${ }^{3}$ and by other experiments.

2. With increasing percentages of carbon the temperature of the change is gradually lowered until with 9 per cent. of carbon it occurs at a minimum temperature of $680^{\circ} \mathrm{C}$. With less quantities of carbon than 9 per cent. the change does not take place at one temperature, but a diminishing amount of $\gamma$ iron (containing the carbon in solid solution) is retained down to $680^{\circ} \mathrm{C}$., where the change is finally completed.

3. A second change occurs in iron and in steels containing less than 45 per cent. of carbon at about $780^{\circ} \mathrm{C}$., which change is marked by an evolution of heat on cooling, a discontinuity in the magnetic properties, and a smaller though clearly marked discontinuity in the tenacity. As to how far this change represents a truly allotropic one is a matter of some doubt, but we do know at least that it is not accompanied by any recrystallization, and for this reason does not enter into the present argument.

4. Iron at temperatures above the allotropic change-point referred to in (I) is capable of holding carbon in solid solution, but in the state which normally exists below the change-point it is incapable (or practically so) of holding it. While it seems probable that it is held in solution in the form of carbide, it is not definitely known whether it is as the carbide $\mathrm{Fe}_{3} \mathrm{C}$ which normally forms when it is thrown out of solution at $680^{\circ} \mathrm{C}$. ; certain experiments ${ }^{6}$ rather tend to the contrary view.

5. The temperature at which amorphous matter, formed by severe overstrain in the cold, commences to recrystallize freely on subsequent heating, and which conversely may be considered as the minimum temperature at which crystallization can occur in an undercooled liquid mass on cooling, 
has been determined for mild steel by Goerens 7 and found to be in the neighbourhood of $500^{\circ} \mathrm{C}$. While a certain amount of crystallization can proceed below this temperature, it is very small when compared with that which proceeds when it is attained. It is doubtful, however, whether any very exact limits can be laid down for the maximum temperature below which the amorphous phase is stable. In the cases where it has been formed by overstrain, it is possible that the actual disturbance from the true crystalline orientation may not be the same in all places where an amorphous structure has been formed, and that therefore a lower temperature is needed to bring about sufficient freedom for the return to the crystalline structure where the disturbance has been slight, than it is where the disturbance has been more severe. The fact that to each temperature to which an overstrained specimen is heated, there corresponds a certain amount of recrystallization (which ceases to increase after a certain length of time at that temperature), rather lends support to such a view.

Again, it has been found impossible entirely to destroy by strain all or even the greater part of the crystalline phase, and therefore we do not know whether crystallization in a purely amorphous mass will begin at the same temperature or with the same freedom as it would in a mass in which a considerable proportion of crystalline entities are still present; it would appear that a purely amorphous mass would be the more stable of the two.

When formed by overstrain in a normally cooled steel, the amorphous matter will be derived chiefly from the a ferrite, since the cementite is practically incapable of plastic deformation. When formed, however, by the breakdown of austenite in a carbon steel the amorphous matter will consist of a mixture of iron and iron carbide, and this mixture may not possess the same viscosity as pure iron. This consideration is referred to below from a somewhat different point of view.

Although it is evident from the above that we cannot state a very exact figure for the maximum temperature of stability of an amorphous iron-iron carbicle solution, yet it is probable that on heating, the stability must become small in the neighbourhood of $500^{\circ} \mathrm{C}$., and that conversely an amorphous solution undercooled below this temperature will possess a certain stability which will rapidly increase on further cooling.

From the experimental data outlined above we see, firstly, that while in the case of pure iron there is a range of about $400^{\circ} \mathrm{C}$. between the temperatures at which the breakdown of the $\gamma$ iron occurs and that at which the recrystallization of $a$ iron becomes difficult, yet in the case of a 9 per cent. carbon steel the range is reduced to less than $200^{\circ} \mathrm{C}$. The probability of retaining by sudden cooling some of the amorphous phase formed by the breakdown of the $y$ structure is therefore much greater with increasing carbon contents. But there is another factor quite apart from this lowering of the temperature of the change-point which causes the presence of carbon to still further promote the retention. Above the change-point the carbide is in solid solution in the austenite, and when this phase breaks down to amorphous the carbide molecules will still remain closely intermingled with those of the $a$ iron. Now, before the amorphous can recrystallize, the two different kinds of molecules have to segregate, since the carbide is insoluble in either $\alpha$ or $\beta$ iron. Such segregation must necessarily be a slow process in an undercooled viscous mass, and rapid cooling may easily allow the minimum temperature of crystallization to be passed before it has had time to take place. A somewhat similar argument to this last has already been advanced by Rosenhain, ${ }^{8}$ but as a reason rather for the retention of austenite than as hindering the crystallization of $\alpha$ iron from amorphous. 


\section{THE PART PLAYED BY THE AMORPHOUS PHASE}

It has been shown by Benedicks 9 that to retain $\gamma$ iron (austenite) in carbon steels pressure, as well as rapid cooling, is necessary ; this fact also follows from the known lowering of the change-points by pressure. Now the change from a crystalline to an amorphous state in metals is, in practically all cases, accompanied by an increase in volume, and in the case under consideration this increase will be still more marked by the fact that crystalline $\gamma$ iron is denser than crystalline $a$, and therefore much more so. than amorphous $a$. Thus when a certain amount of $\gamma$ iron has broken down to amorphous it will occupy more space in the mass and will tend to compress. the $\gamma$ iron that has not already changed and therefore to prevent its doing so. Quenching therefore, besides preventing the crystallization of the amorphous mixture formed by the breakdown of the austenite, may at the same time preserve a certain amount of that austenite unchanged. The more severe the quenching, the more austenite retained, since the rapid increase in rigidity of the amorphous mass as it cools will enable it to exert its pressure more effectively.

The theory of the hardening of steels outlined above depends upon two points: (I) that the temperature of the change-point is lowered by quenching, and (2) that during a normal allotropic change there must exist a temporary amorphous state. The influence of the first point has already been advanced by Grenet, ${ }^{\text {to }}$ the second now shows how this lowering of the changepoint may bring about hardness, since it involves the retention of the amorphous state, and such a state is known to be one of particular hardness in practically all metals.

We may now pass to the consideration of how the theory agrees with, and is consistent with, the known physical properties and microstructure of carbon steels hardened by quenching, and of certain alloy steels which possess a similar hard structure even when slowly cocled.

Physical Properties.-The physical properties of a steel hardened by quenching and of the same material hardened by overstrain are in many ways similar, and strongly point to the idea that a similar form of structure is. present. Thus in both cases it has been found that the hard metal possesses a distinctly lower specific gravity than the same samples when annealed. Among other properties in which overstrained and quenched steels vary in a similar manner from the annealed metal may be cited the possession of high retentivity, coercive force, and coefficient of hysteresis. The fact pointed out above, that in overstrained steel the carbide and the iron molecules. will remain segregated, while in a quenched steel they will be very intimately mixed in solution, may prevent the similarity from being too close.

Microstructure.-The typical micro-constituent of a quenched steel is martensite, which may-according to the carbon contents and the rate of quenching - vary from an almost structureless mass to a strongly marked duplex structure of interlacing needles, the latter type being characteristic of high carbon contents and rapid cooling. According to the present theory the duplex structure consists of the two phases $\gamma$ iron (austenite) and an amorphous solution of $a$ iron and carbide; the form in which the two phases occur together being due, as advanced above, to the tendency of the austenite crystals to break up most readily along their gliding planes. Conditions which produce an almost structurcless martensite are those under which, whiie practically all austenite has been able to change to amorphous, yet little or no recrystallization of the amorphous has been possible. Such conditions occur in practice when samples are quenched from not too high a temperature; in such a state a steel should possess its maximum hardness combined with a freedom from internal stresses, and therefore be in the most 


\section{IN THE HARDENING OF STEELS}

favourable condition from a practical point of view. Rapid quenching from too high temperatures, which results in the retention of much of the austenite, will produce a less satisfactory result, since besides the fact that austenite is known to be less hard than the amorphous solution, its presence implies internal stresses and liability to hardening cracks and spontaneous fractures. These points are of course well known to all who have dealt with the hardening of steels.

Alloy Steels.-The present theory of hardening applies with special aptness to the case of certain alloy steels, which acquire a hard structure even when slowly cooled. Alloy steels, of which the nickel-carbon series may be taken as a typical examples, are found when normally cooled to possess three different types of structure according to the percentage of these elements which they contain, viz. (a) pearlitic, (b) martensitic, or $(c)$ austenitic. The increasing percentages of the added elements produce a progressive lowering of the change-point, and it has been found that the structure of type (a) occurs in all steels in which the change-point lies above about $400^{\circ} \mathrm{C}$.; of type $(b)$ in all the steels in which the change-point lies between about $400^{\circ} \mathrm{C}$. and atmospheric temperature; and of type $(c)$ in all the steels in which the change-point lies below atmospheric temperature. The last type, however, become martensitic if undercooled to below their change-point. These results show that in all the steels in which a change-point occurs below $400^{\circ} \mathrm{C}$. -a temperature which corresponds very approximately to that below which we have seen the amorphous phase is stable-possess a structure and a hardness which is similar to that of a quenched carbon steel. The present theory exactly accounts for such a phenomenon, since it states that when the breakdown from the $\gamma$ condition takes place at a temperature at which the amorphous phase is stable, a hard amorphous structure will persist. The fact that the partial change to amorphous can, and does, occur in these steels in spite of low temperature is evidence that the breakdown of a crystalline condition is possible in spite of low temperature.

Two further points may be emphasized in regard to the bearing of the present theory upon the normal austenitic or martensitic structure of alloy steels.

(1) Cold work applied to an austenitic alloy causes a new and intensely hard phase to appear along the slip planes of the $\gamma$ crystals, ${ }^{11,12}$ and this phase has been shown to possess all the characteristics of martensite. The quantity produced by a given amount of deformation is out of all proportion to the amount of amorphous phase formed by an equal amount of deformation in other metals. If we consider, as the present theory suggests, that the crystals in an austenitic steel are in most cases within less than $100^{\circ} \mathrm{C}$. of a temperature at which they spontaneously change to an amorphous state, it is conceivable that when once a certain breakdown occurs along a slip plane due to the deformation, it may proceed with much greater freedom than it would in the case of an ordinary metal in which no such spontaneous change is involved.

(2) The present theory offers an explanation of the irreversible nature of the changes which occur in alloy steel, i.e. that the change from the austenitic to the martensitic structure takes place at a much lower temperature than the reverse change on heating. It suggests that while the change from the $\gamma$ state to the amorphous on cooling is independent of low temperature, yet the completion of the change, which would be the recrystallization of the amorphous to $\alpha$, is prevented by it, and that in consequence the amorphous phase persists. The reverse change of amorphous to $\gamma$ crystals on heating will be prevented by precisely the same cause, viz. that crystallization of any 


\section{THE PART PLAYED BY THE AMORPHOUS PHASE}

kind cannot take place until the viscosity has sufficiently diminished to allow of the necessary molecular movements. It has been found that about $400^{\circ} \mathrm{C}$. is the minimum temperature at which the change on heating occurs in any of the series of irreversible alloys, and this temperature may therefore be assumed to be the maximum at which an amorphous solution of iron, nickel, and carbide is stable. It will be remembered that it is the same as the temperature at which the change occurs in the alloys which first show a martensitic structure on normal cooling.

Reference may be made at this point to the "high speed" steels. The recrystallization either on heating or cooling of an amorphous solution will depend particularly upon its viscosity, and the viscosity itself upon the temperature and the composition of the mixture. In high-speed steels we have the two properties, firstly that of air hardening, and secondly that of the stability of the hard structure to high temperatures. The latter property appears to be given to the steels by elements such as chromium, tungsten, etc., which are known to form definite carbides. It appears probable that these carbides act by greatly increasing the viscosity of an amorphous solution containing them, and so allow the amorphous state to remain stable at much higher temperatures.

Tempering.-The tempering of hardened steels will, according to the present theory, consist in the progressive crystallization of the amorphous solution formed by the quenching, but the process is possibly rather more complicated than would at first sight appear to be the case, since, from the experiments of Campbell and Haskins (referred to on page 242) it appears to be accompanied by polymorphic changes in the composition of the carbide. The first segregation of an undercooled solution of $a$ iron and iron carbide would, however, agree very well with Benedick's theory of troostite as a colloidal system. It seems possible that the segregation of the two different kinds of molecules might advance some way before an actual crystalline structure, however fine, is formed; in such a condition the structure would correspond to that of a true liquid colloidal solution. We can in this way distinguish between the two phases troostite and sorbite, the former being a partially segregated amorphous mixture of $a$ iron and iron carbide, while the latter is an extremely fine crystalline mixture of the same constituents.

It may be noted that the temperature at which a steel loses all the hardness which it has acquired by quenching agrees very approximately with that at which it loses any hardness acquired by overstrain.

Summary. - The conclusions arrived at in the present paper may be summarized as follows :-

The hard structure which can be produced in carbon steels by quenching and in certain alloy steels by normal cooling, is due to the presence of a hard amorphous solution of $\boldsymbol{a}$ iron and iron carbide, which solution may be compared to Beilby's amorphous phase formed by overstrain.

To explain the formation of this amorphous phase the author advances a theory that the passage of a substance from one allotropic modification to another of different crystalline form involves the temporary formation of an amorphous state, corresponding to the liquid phase of the modification about to be formed. In iron such a change occurs at $\mathrm{Ar}_{1}$; and if, due to sudden cooling or to the presence of certain alloyed elements, the change-point is lowered to a temperature below that at which crystallization in the viscous mass becomes difficult, then the amorphous form will be retained in a metastable form in the cold. 


\section{IN THE HARDENING OF STEELS}

The infuence of carbon in enabling a hard amorphous structure to be retained by quenching is twofold, viz.:-

(I) As above, by its action in lowering the temperature of the change point ; and

(2) In a more essential manner, by the fact that-owing to their mutual insolubility in the solid state-segregation movements of both the $a$ iron and the iron carbide molecules must take place in the amorphous solution formed at $\mathrm{Ar}_{3}$, before either can assume a crystalline formation.

\section{REFERENCES.}

I Rosenhain and Ewen: "Intercrystalline Cohesion in Metals," $\widetilde{f}$ ournal of the 1nst. of Mctals, No. 2, 1912, vol. viii.

${ }^{2}$ Rosenhain and Ewen: "Intercrystalline Cohesion of Metals," second paper, Fournal of the Inst. of Metals, No. 2, I913, vol. x.

3 Rosenhain and Humfrey: "The Tenacity, Deformation, and Fracture of Soft Steel at High Temperatures," Fournal of the Iron and Stcel Inst., r9I3, No. I.

4 Humfrey: "The Influence of Intercrystalline Cohesion upon the Mechanical Properties of Metals," Iron and Steel Inst., Carnegie Mcmoirs, I9I3.

5 Humfrey : "The Intercrystalline Fracture of Iron and Steel," Iron and Steel Inst., Carnegie Memoirs, I913.

${ }^{6}$ Campbell and Haskins : "Effects of Heat Treatment on the Colorimetric Test for Carbon in 0.32 per cent. Carbon Steel," Fournal of the Iron and Steel Inst., 1913, No. 2.

7 Goerens: "On the Influence of Cold Working on the Properties of Iron and Steel," Iron and Steel Inst., Carnesie Memoirs, I9II.

8 Rosenhain: "Recent Advances in the Metallography of Steel," Staffordshire Iron and Steel Inst., 1910.

9 Benedicks: "The Cooling Power of Quenching Velocities, etc.," Fournal of the Iron and Steel Inst., I908, No. 2.

1o Grenet: "The Transformations of Steel within the Limits of the Temperatures Employed in Heat Treatment," Fournal of the Iron and Stecl Inst., I9II, No. 2.

Ir Carpenter, Hadfield, and Longmuir : "On the Properties of a Series of IronNickel-Manganese-Carbon Alloys," Seventh Report of the Alloys Research Committee, Institute of Mechanical Engineers, November I7, 1905.

12 Rosenhain : "Deformation and Fracture of Iron and Steel" Fournal of the Iron and Sted Inst., I906, No. 2. 